\title{
MIXTURE-ORDER DESIGN OF GPS NETWORKS BASED ON GENETIC ALGORITHMS
}

\author{
M. F. Sobeih ${ }^{1}$, M. I. Doma ${ }^{1}$, and A. F. El Shoney ${ }^{2}$ \\ 1. Civil Engineering Departement, Faculty of Engineering, Minufiya University ,Egypt \\ 2. Survey Research Institute, National Water Research Center, \\ Ministry of Irrigation, Egypt E-mail: as_amien@yahoo.com
}

\begin{abstract}
Today, most geodetic networks are being measured by differential Global Positioning System (GPS). Nevertheless, producing optimal baseline configuration and optimal baseline weights "Mixture-Order Design" in designing stage are necessary pre-requisites for an accurate geodetic GPS network. In this paper, a very efficient method based on the concept of the genetic algorithms metaheuristic technique is developed for providing an efficient solution for Mixture-Order Design problem. In the present study, the proposed optimization modeling is applied only to determine which of the baselines could be deleted while adhering to the prescribed precision of the network, which may result in significant savings on the cost of the project.

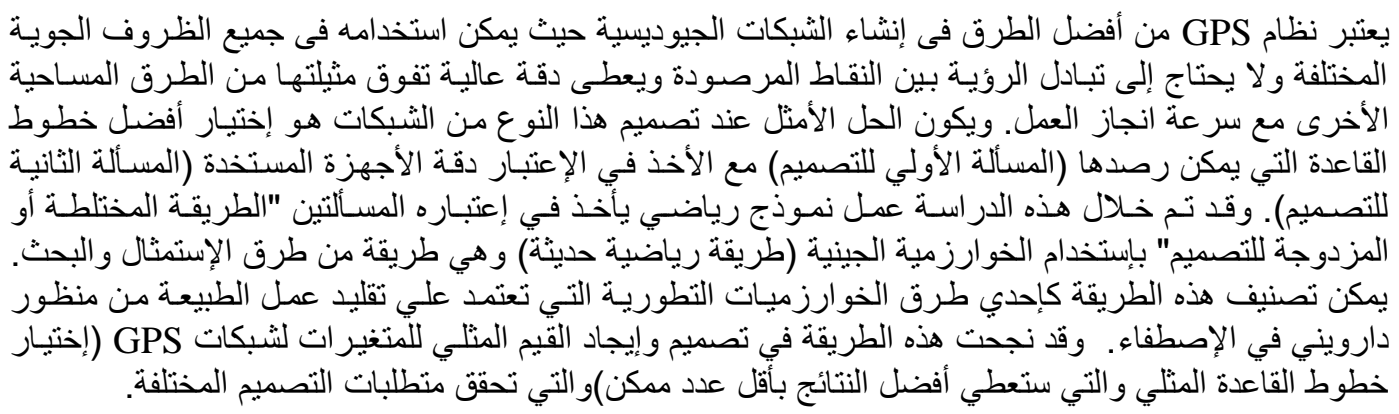

Keywords: GPS Networks, Genetic Algorithms, Mixture-Order Design Problem, Optimization

\section{INTRODUCTION}

For hundreds of years, surveyors used to rely on optical instruments and physical measuring devices (e.g. tape measures or chains). Optical instruments require direct line of site from the instrument to a target. Measuring tapes or chains require that the survey crew physically pass through all intervening terrain to measure the distance between two points. Surveying methods have undergone a revolutionary change over the last three decades with the deployment of the satellite navigation systems (e.g. Global Positioning System GPS). On the other hand, satellite systems equipment is very expensive compared with other methods, and this becomes crucial when the amount of work increases for large projects. A GPS network is distinctly different from a classical survey network in that no inter-visibility between stations is required. In GPS surveying, after defining the locations of the points for an area to be surveyed, GPS receivers will be used to map this area by creating a network of these coordinated points. These points, control stations within the context of surveying, are fixed on the ground and located by an experienced surveyor according to the nature of the land and the requirements of the survey. (Elshouny A.F., 2008)

The main purpose of this study is to establish a new method based on genetic algorithms, which are inspired from population genetics, for optimizing the GPS surveying network problem (optimal baseline configuration and optimal baseline weights). The paper is organized as follows: A general framework is described for the optimization problem. Then, the search strategy of the proposed GAs technique, its structural elements and genetic operators are explained. Subsequently, a numerical case study has been applied to asses the performance of the proposed technique. Finally the conclusion and discussion of possible directions for future research are outlined.

\section{DEFINITION OF THE OPTIMAZATION PROBLEM}

Historically, the design problems were defined and initially dealt with in the 1970s and the 1980s. 
The first studies-pioneered by Grafarend (1974) and Baarda (1973)- were collected in the classic book by Grafarend and Sanso (1986).

Optimization problem is usually classified into different orders. Determination of the optimum geometric design for a geodetic network, one of the classical design problem (FOD). There are also zeroorder, second-order, third-order and finally mixture (combined) order design problems. The zero-order design (ZOD), also called the datum problem, aims to datum definition: which restrictions are to be imposed on the model to find a solution that is free from the influence of fixed-coordinate in accuracies. Hence, in the ZOD datum points are the variables. The First-Order Design, also called the configuration problem, optimizes station positions and the observations to be made, this method aims at achieving mainly two items. First, it aims to achieve the optimal location of points of the network or the optimal geometry of the network. Second, it aims to achieve an optimal observational plan of the considered network. This means to determine what is to be measured, the type of measurements and its distribution all over the network. The Second-Order Design problem (SOD), also called the weight problem, aims to design the observations weights so that the solution is able to accomplish prescribed precisions. The variable in this problem is the observations' weight matrix. The Third-Order Design problem (THOD), also called the improvement problem, deals with optimal network densification. Finally, The Mixture Order Design problem (MOD), also called the combined design problem, where both the (FOD) and the (SOD) problems have to be optimally solved simultaneously. The variables in this problem are the observations' design matrix and the observations' weight matrix. In the current study, we concentrate on the mixture-order design problem and its solution. ( Berné J.L. et al., 2004).

By considering the elements of the Least-square adjustment as free and fixed parameters, this classification can be characterized as shown in table (1).

The optimal design problem to be considered here for a GPS relative survey campaign in this paper is to determine optimal baseline configuration and optimal baseline weight distribution of a GPS network (MOD), that will satisfy the required criteria with the minimum cost and ensure maximum homogeneity and isotropy conditions at the end of the optimization procedures. (Teke K. et al., 2008).

\subsection{Mixture Optimization Problem Formalization}

GPS network can be defined as a set of stations, co-ordinate by a series of sessions formed by placing receivers on the stations. (Saleh H.A. et al., 2001).
Table 1, Classification of the optimization design problem (Doma, 2004)

\begin{tabular}{|c|c|c|c|}
\hline \multicolumn{2}{|c|}{ DESIGN PROBLEM } & FIXED & FREE \\
Order & Item to be optimized & PARAMETERS & PARAMIETERS \\
\hline ZOD & Datum of Geodetic Network & $\mathrm{A}, \mathrm{P}$ & $\mathrm{Qx}$ \\
\hline FOD & $\begin{array}{c}\text { Geodetic Network } \\
\text { Configuration }\end{array}$ & $\mathrm{P}, \mathrm{Qx}$ & $\mathrm{A}$ \\
\hline SOD & Observations Weights & $\mathrm{A}, \mathrm{Qx}$ & $\mathrm{P}$ \\
\hline THOD & Densifications & $\mathrm{Qx}$ & $\mathrm{A}, \mathrm{P}$ (partially) \\
\hline $\begin{array}{c}\text { MIXTURE } \\
\text { (MOD) }\end{array}$ & $\begin{array}{c}\text { Configuration and } \\
\text { Observations Weights }\end{array}$ & $\mathrm{Q} \times$ & $\mathrm{A}, \mathrm{P}$ \\
\hline
\end{tabular}

The variance-covariance matrix of station coordinates is written as (Doma, 2004):

$$
\mathbf{C}_{\mathrm{x}}=\sigma_{\mathrm{o}}^{2}\left(\mathbf{A}^{\mathrm{T}} \mathbf{P} \mathbf{A}\right)^{-1}
$$

Since in the above equation matrix $\mathbf{A}$ is a constant matrix, matrix $\mathbf{C}_{\mathbf{x}}$ consists of nonlinear functions of only observational weights $\mathbf{p}_{\mathrm{i}}(\mathrm{i}=1,2, \ldots$ ,n), which are, therefore, the basic unknown parameters to be optimally solved for at the design stage of the network. Given a set of approximate values for observational weights, matrix $C_{x}$ can be approximated by Taylor series of linear form (Kuang S.L., 1994):

$$
\mathbf{C}_{\mathbf{x}}=\mathbf{C}_{\mathbf{x}}^{\mathbf{o}}+\sum_{\mathrm{i}=1}^{\mathrm{n}} \frac{\partial \mathbf{C}_{\mathbf{x}}}{\partial \mathbf{p}_{\mathrm{i}}} \Delta \mathbf{p}_{\mathrm{i}}
$$

where:

$$
\begin{aligned}
\mathbf{C}_{\mathrm{X}}^{\mathrm{o}} & =\left.\sigma_{\mathrm{o}}^{2}\left(\mathbf{A}^{\mathrm{T}} \mathbf{P} \mathbf{A}\right)^{-1}\right|_{\mathbf{P}^{\mathrm{o}}} \\
\frac{\partial \mathbf{C}_{\mathrm{x}}}{\partial \mathbf{p}_{\mathrm{i}}} & =\left.\sigma_{0}^{2}\left\{-\left(\mathbf{A}^{\mathrm{T}} \mathbf{P A}\right)^{-1}\left[\mathbf{A}^{\mathrm{T}} \frac{\partial \mathbf{P}}{\partial \mathbf{p}_{\mathrm{i}}} \mathbf{A}\right]\left(\mathbf{A}^{\mathrm{T}} \mathbf{P A}\right)^{-1}\right\}\right|_{\mathbf{P}_{0}}
\end{aligned}
$$

$\mathbf{P}^{\mathbf{0}}$ is the initial observation weight matrix, and $\Delta \mathrm{p}_{\mathrm{i}}(\mathrm{i}=1,2, \ldots, \mathrm{n})$ are the improvements, which are to be optimally solved for, to the initially given weights. Assuming that no correlation between observations exists, the partial derivatives of the weight matrix $\mathrm{P}$ with respect to the individual observational weight $\mathrm{p}_{\mathrm{i}}$ are given below:

$$
\begin{aligned}
\frac{\partial P}{\partial p_{i}} & =\operatorname{diag}\left(E_{j}\right) \text { with } E_{j}=\left\{\begin{array}{ll}
I_{3} & \text { if } j=i \\
0_{3} & \text { if } j \neq i
\end{array} \quad(j=1,2, \ldots . ., n)\right.
\end{aligned}
$$

$\mathbf{I}_{\mathbf{3}}$ and $\mathbf{0}_{\mathbf{3}}$ the 3 by 3 identity and zero matrices, respectively.

Similarly, criterion matrix $\mathrm{C}_{S}$ is used as the precision criteria in this case; the design problem seeks an optimal configuration matrix $\mathrm{A}$ and weight matrix $P$ such that $C_{S}$ can be best approximated by $\mathrm{C}_{\mathrm{x}}$. The precision function in this case is:

$$
\left\|\mathbf{C}_{\mathbf{x}}-\mathbf{C}_{\mathbf{S}}\right\|=\min \quad \text { (optimal design) }
$$


$\operatorname{vec}\left(\mathrm{C}_{\mathrm{x}}\right) \leq \operatorname{vec}\left(\mathrm{C}_{\mathrm{S}}\right) \quad$ (precision control) where,

vec: The operation produces a vector by staking the column of a quadratic matrix under another in a single column.

Finally, by linearazation of the precision criteria, we can reformulate precision criteria in a compact matrix and vector form (Doma, 2008):

$$
\begin{array}{ll}
\|\mathbf{V} \cdot \mathbf{w}-\mathbf{u}\|=\min & \text { (optimal design) } \\
\mathrm{V}_{1} \cdot \mathrm{w}-\mathrm{u}_{1} \leq 0 & \text { (precision control) }
\end{array}
$$

Denote:

$$
\begin{aligned}
& \mathbf{u}=\operatorname{vec}\left(\mathbf{C}_{\mathrm{S}}\right)-\operatorname{vec}\left(\mathbf{C}_{\mathbf{x}}^{\mathbf{o}}\right) \\
& \mathbf{V}=\left(\operatorname{vec}\left(\frac{\partial \mathbf{C}_{\mathbf{x}}}{\partial \mathbf{p}_{1}}\right) \operatorname{vec}\left(\frac{\partial \mathbf{C}_{\mathbf{x}}}{\partial \mathbf{p}_{2}}\right) \ldots \operatorname{vec}\left(\frac{\partial \mathbf{C}_{\mathbf{x}}}{\partial \mathbf{p}_{\mathrm{n}}}\right)\right) \\
& \mathbf{w}=\left(\begin{array}{lll}
\Delta \mathbf{p}_{1} & \Delta \mathbf{p}_{2} & \ldots \Delta \mathbf{p}_{\mathbf{n}}
\end{array}\right)^{\mathrm{T}} \\
& \mathbf{V}_{1}=\left(\mathbf{I}_{\mathbf{u}} \Theta \mathbf{I}_{\mathbf{u}}\right)^{\mathrm{T}} \mathbf{V}, \quad \mathbf{u}_{1}=\left(\mathbf{I}_{\mathbf{u}} \Theta \mathbf{I}_{\mathbf{u}}\right)^{\mathrm{T}} \mathbf{u}
\end{aligned}
$$

$\boldsymbol{\Theta}$ : The same previous definition

\subsection{Genetic Algorithms}

Among the set of search and optimization techniques, the development of Evolutionary Algorithms (EA) has become very important in the last decade. EAs are a set of modern met heuristics used successfully in many applications with great complexity and success on solving difficult problems. EAs form a subset of Evolutionary computation (EC) which is the general term for five main computational techniques based on principles of biological evolution, such as natural selection and genetic inheritance. These five main techniques are Genetic Algorithms (GA) (Holland 1975), Genetic Programming (GP) ( Koza 1992, 1994 ), Evolution Strategies (ES) ( Recheuberg 1973 ), Evolutionary Programming (EP) ( Forgel et al ,1966 ) , and learning Classifier Systems (CS) (Holland 1976) . Genetic Algorithms (GAs) are the most popular technique and most active subset of evolutionary computation.(Sivanandam et al.,2008). Figure 1. shows the different families of evolutionary algorithms.

Genetic Algorithms (GAs) are general purpose heuristic search algorithms that mimic the evolutionary process in order to find the best fit solutions. They have received growing interest due to their simplicity as algorithms and their ability to discover good solutions quickly for complex searching and optimization problems. The Algorithms are particularly suitable for solving complex optimization problems and for applications that require adaptive problem-solving strategies, because the Algorithms are parallel in nature and they can offer a number of possible solutions. Their potential applications are numerous. They have been employed in a wide range of applications including but not limited to: optimization of functions with linear and nonlinear constraints, the traveling salesman problem, machine learning, parallel semantic networks, simulation of gas pipeline systems, problems of scheduling, web search, software testing, and financial forecasting. Similar to the other heuristic search Algorithms, the performance of a GA can be measured by the cost, time, and the quality of the solution.

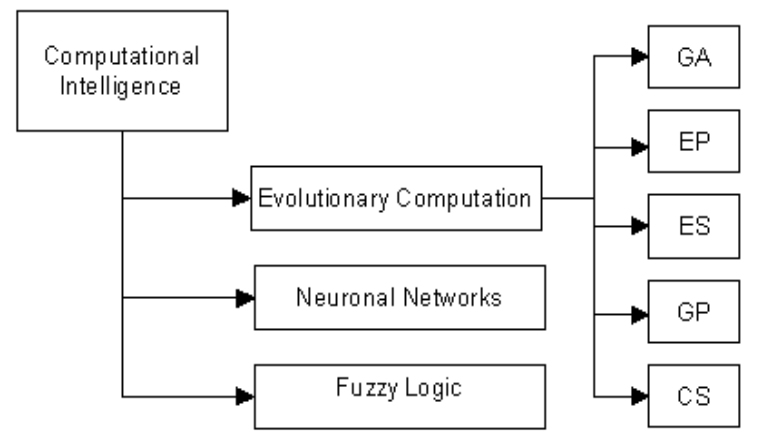

Fig. 1 Location of the different families of evolutionary algorithms

* Comparison with other optimization

Genetic algorithm differs from conventional optimization techniques in the following fundamental differences: (Sivanandam et al., 2008).

1- GAs use probabilistic transition operates while conventional methods for continuous optimization apply deterministic transition operates i.e., GAs does not use deterministic rules.

2- GAs use fitness function for evaluation rather than derivatives. As a result, they can be applied to any kind of continuous or discrete optimization problem. The key point to be performed here is to identify and specify a meaningful decoding function.

3- GAs operate with coded versions of the problem parameters rather than parameters themselves i.e., GAs work with the coding of solution set and not with the solution itself.

4- Almost all conventional optimization techniques search from a single point but GAs always operate on a whole population of point (strings) i.e., GAs use population of solutions rather than a single solution from searching. This plays a 
major role to the robustness of genetic algorithms. It improves the chance of reaching the global optimum and also helps in avoiding local stationary point.

5- The objective function in GAs can be numerical or logical. This is because the variables are coded. For example, image processing, pattern recognition or vehicle routing control problems may contain logical objective terms that cannot be tackled by conventional methods. This gives great flexibility to a wide range of applications.

6- Unlike GAs, conventional optimization deals with constraints as separate independent statements. GAs, on the other hand, tackle constraints by placing a penalty in the fitness function. To illustrate this, Deb used the following nonlinear programming problem (1996).

$$
\begin{aligned}
& \text { Minimize } f(x) \text { subject to } \\
& G_{j}(x) \geq 0 \quad \text { for } \quad j=1,2, \ldots ., k \\
& H_{k}(x)=0 \quad \text { for } k=1,2, \ldots, m \\
& x_{i \min } \leq x_{i} \leq x_{i \max } \quad \text { for } i=1,2, \ldots ., n
\end{aligned}
$$

With GAs, the above constraints can be incorporated directly into the fitness function. For this example, the constraints can be addressed in a single statement within the objective. This simplifies the problem into an unconstrained, "penalized" fitness (objective) function:

$$
p(x)=f(x)+\sum_{j=1}^{k}\left[G_{j}(x)\right]^{2}+\sum_{k=1}^{m}\left[H_{k}(x)\right]^{2}
$$

If a solution violates a constraint, then the fitness function is penalized by a factor proportional to the square of the constraints (Deb K., 1996).

The basic steps of genetic algorithms will be described as following:

$$
\mathbf{C}_{\mathrm{x}}=\sigma_{\mathrm{o}}^{2}\left(\mathbf{A}^{\mathrm{T}} \mathbf{P} \mathbf{A}\right)^{-1}
$$

\section{* Design objective}

The optimization design objective needs in this paper can be formulated as following:

\section{- Encoding problem}

The basic element of the genetic Algorithms is the chromosome which contains the variable information for each individual solution to the problem. The chromosomes should be encoded in a way that offers information about the solutions that they represent the encoding process is a central factor in the success of a genetic algorithm. The main types of encoding process: Binary encoding, Octal encoding, Hexadecimal encoding, Permutation encoding (Real Number Coding) and Value encoding. (Riesberg L., 2003).

\section{- $\quad$ Initial population}

The Genetic Algorithms sequence begins with the creation of an initial population of individual the population size depends on the nature of the problem, but typically contains several hundreds or thousands of possible solutions. Traditionally, the initial population is generated randomly, covering the entire range of possible solutions (the search space), occasionally, it can be generated heuristicly by using prior knowledge of possibly good solutions. (PonceCruz P. et al., 2010).

\section{- Evaluation of fitness function}

Choosing an appropriate evaluation function is an essential step for successful application of GAs to any problem domain. Fitness function is a particular type of objective function that measures the optimality of the solution and the explicity of the performance of each chromosome in the population. The fitness of a chromosome depends on how well that chromosome closes to the optimal solution. The higher the fitness value, the closer is the solution to the optimal solution. (Melanie M., 1999).

\section{- $\quad$ Termination criteria}

The generational process is repeated until a termination condition has been reached. The commonly used termination conditions are (Saleh et al., 2004):

1. Stall generations: the algorithms stop if there is no improvement in the objective function for a sequence of generations of the length of stall generations.

2. Fitness limit: the algorithms stop when the value of the fitness function is less than or equal to fitness limit.

3. Generations: the algorithms stop when reach the number of selected generations.

4. Stall time limit: the algorithms stop if there is no improvement in the objective function during an interval of time in seconds equal to stall time limit.

5. Time limit: the algorithms stop after running for an amount of time in seconds equal to time limit.

\section{* Selection}

During each successive generation, a proportion of the existing population is selected to breed a new generation. The selection operator allocates more copies to solutions with better fitness values and thus imposes the survival of-the-fittest mechanism on the candidate solutions. The main idea of selection is to prefer solutions to worse ones; without a good selection method, the GAs may wander away from its target problem and create random chromosomes that have no bearing on the problem whatsover. (Fidanova S. et al., 2010). 
There are three commonly used selection methods as described below: (Weise T., 2009).

a) Roulette wheel selection

Roulette wheel selection is one of the traditional GAs selection methods. The individuals are selected by a probability proportional to the normalized fitness. This means that all fitness measures have to be gathered, summed and normalized, before being distributed back to all the individuals again. This method is illustrated exemplarily in Figure (2).
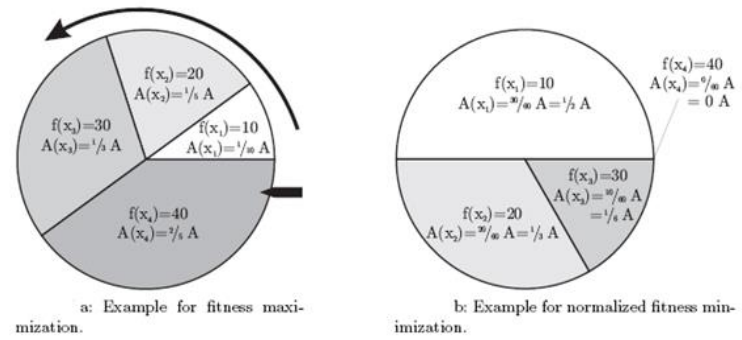

Fig. 2 Examples for the Idea of Roulette Wheel Selection

\section{b) Ranking selection}

In ranking selection, the probability of an individual to be selected is proportional to its position (rank) in the stored list of all individuals in the population. Using the ranking eliminates the weaknesses of proportionate selection. The selection probability is linearly assigned to all individuals according to their rank. (Sivanandam et al., 2008).

\section{c) Tournament selection}

Two or more individuals are selected on random from the population. These individuals compete and compare their fitness values, leaving the one with the best fitness as the selected individuals. This method is more efficient and leads to an optimal solution. (Ekland S.K., 2004).

\section{* Reproduction}

The genetic operation of reproduction is based on the Darwinian principle of reproduction and survival of the fittest. In reproduction operation, an individual is probabilistically selected from the population based on its fitness and then the individual is copied, into the next generation of the population. The Selection is done in such a way that the better an individual's fitness the more likely it is to be selected. An important aspect of this probabilistic selection is that every individual however poor its fitness, has some probability of selection. (Balasubramaniam P. et al., 2009).

\section{* Crossover}

Crossover, or recombination, is an important random operator in GAs that combines (mates) two chromosomes (parents) to produce new chromosomes (offsprings). The main idea behind crossover is that the new chromosome maybe better than both of the parents if it takes the best characteristics from each of the parents. Crossover maybe performed using one of the following methods: One-point crossover, two-point crossover and uniform crossover.
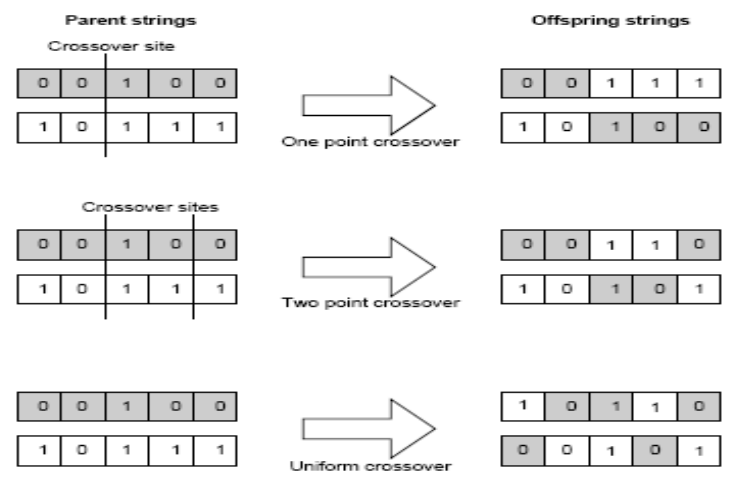

Fig. 3 Illustration of one-point, two-point, and uniform crossover operators

\section{* Mutation}

Mutation is another important component in GAs. The mutation process is very simple. It is the process which deals with the most fit solutions by changing some of binary digits for individuals by random way. The user specifies a mutation probability, which is generally much lower than the crossover probability. The main goal of this operator is to have diversity in the population. ( Chih - Hung et al., 2008 ).
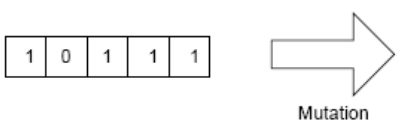

\begin{tabular}{|l|l|l|l|l|}
\hline 1 & 0 & 1 & 0 & 1 \\
\hline
\end{tabular}
Fig. 4 Illustration of bitwise mutation operator

\section{* Elitism}

It is the final step of genetic algorithms steps that must be performed on the new generation is elitism. Elitism is the process of selecting the fittest solution from any generation and transferring it to the new generation to be sure that the fitness can't be less than fitness in the previous generations.

\section{COMPUTER PROCESSING}

Using the Pc-Matlab language with a personal computer and the proposed mathematical model based genetic algorithm, a new program has been devoted to solve of Mixture-Order Design problem of the GPS survey planning. This program has been designed, written and tested by others. Figure (5) shows a flowchart of the new program, which will solve the proposed approach. 
ERJ

Engineering Research Journal

Faculty of Engineering

Minoufiya University

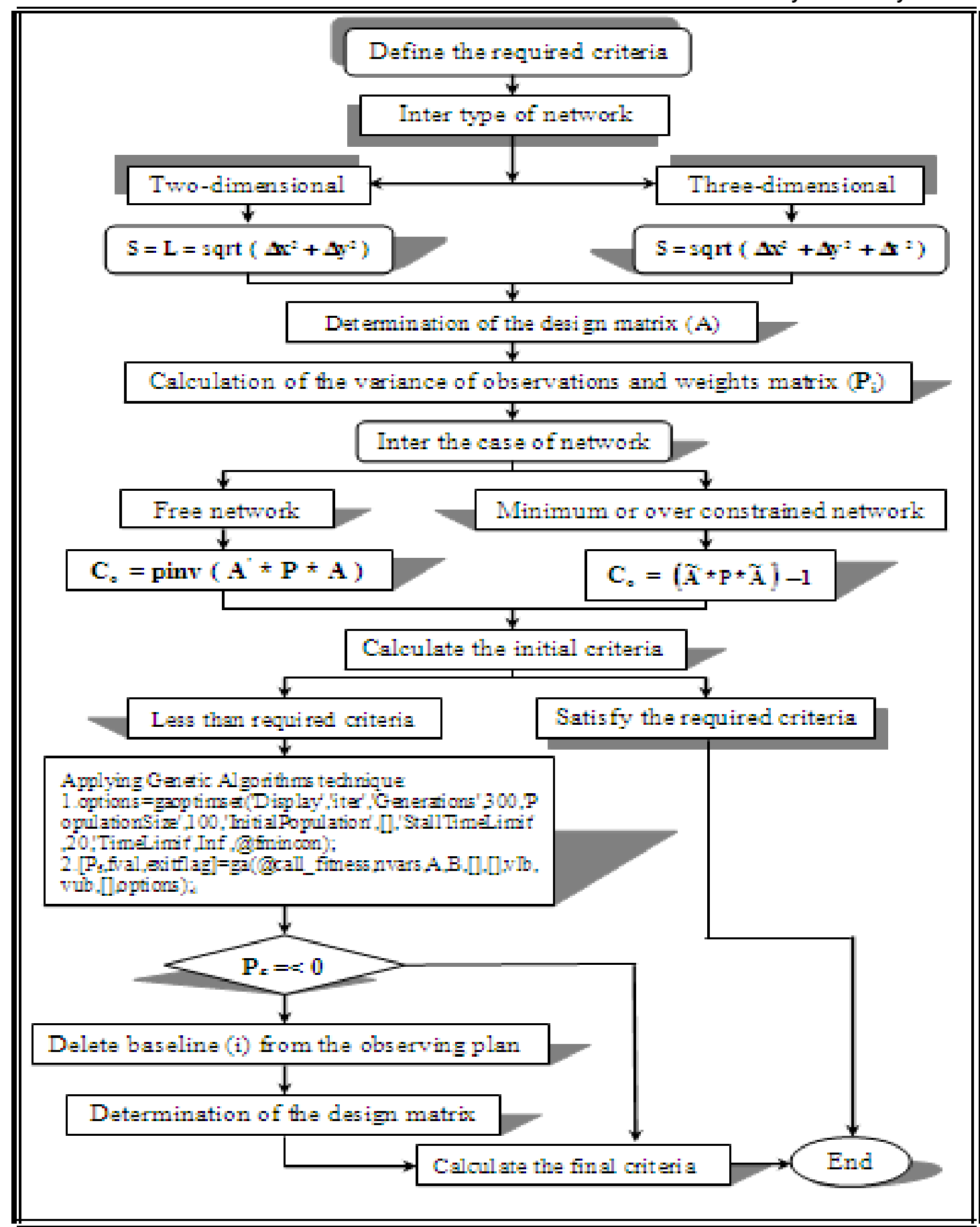

Fig. 5 Flowchart of new program 


\section{NUMERICAL STUDY}

The proposed mathematical model is applied based on genetic algorithms as described above to the problem of optimizing GPS geodetic network design with new MATLAB program. Assuming that we have a three-dimensional network of 12 stations to be positioned using the GPS relative positioning technique as shown in Figure (6). The simulated approximate coordinates of net points are listed in Table (2).

Assume that, points 1 and 8 are fixed to provide the datum of the network and the selected GPS receivers can allow for a baseline to be determined with the following precision: $\sigma_{s}^{2}=(5)^{2} \mathbf{m m}+(1 p p m . S)^{2}$, where $S$ is the distance computed from the approximate coordinates. The optimization is done under precision criteria; all the standard deviations of the coordinate components must be less than $25 \mathrm{~mm}$ ( required criteria ).

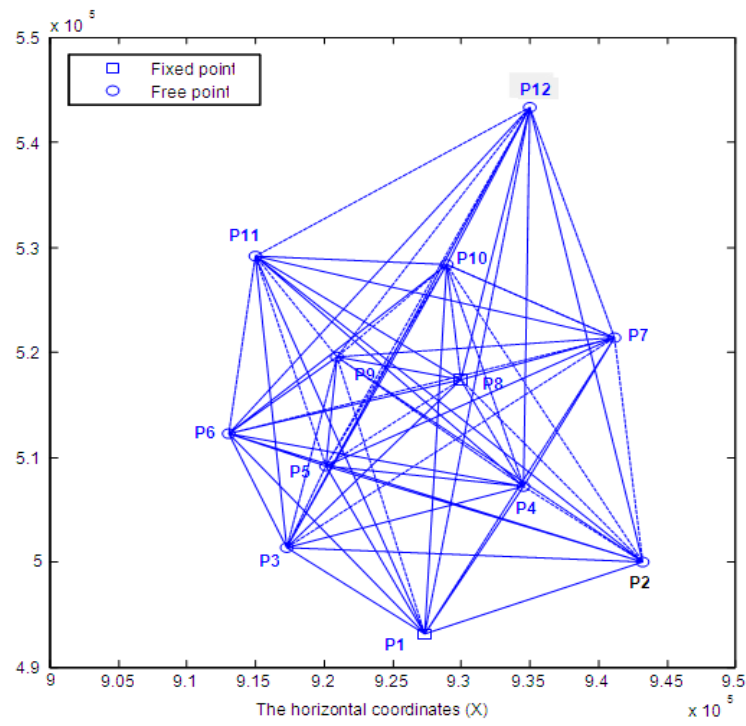

Fig. 6 All possible combinations of baselines

Table 2, The simulated approximate coordinates of network points

\begin{tabular}{||c|c|c|c||}
\hline \multirow{2}{*}{ Point } & \multicolumn{2}{|c|}{ Simulated coordinates of netpoints } \\
\cline { 2 - 4 } & $\mathbf{x}(\mathbf{m})$ & $\mathbf{y}(\mathbf{m})$ & $\mathbf{z} \mathbf{( m )}$ \\
\hline $\mathbf{P}_{1}$ & 927311.02 & 493150.02 & 2.348 \\
\hline $\mathbf{P}_{\mathbf{2}}$ & 943222.01 & 500022.11 & 7.162 \\
\hline $\mathbf{P}_{\mathbf{3}}$ & 917333.01 & 501332.10 & 3.559 \\
\hline $\mathbf{P}_{4}$ & 934579.734 & 507176.193 & 15.513 \\
\hline $\mathbf{P}_{\mathbf{5}}$ & 920144.220 & 509110.321 & 3.201 \\
\hline $\mathbf{P}_{6}$ & 913053.811 & 512239.973 & 2.875 \\
\hline $\mathbf{P}_{\mathbf{7}}$ & 920962.902 & 519505.389 & 25.36 \\
\hline $\mathbf{P}_{8}$ & 929931.01 & 517405.598 & 16.59 \\
\hline $\mathbf{P}_{\mathbf{9}}$ & 941164.684 & 521424.341 & 29.36 \\
\hline $\mathbf{P}_{10}$ & 928991.34 & 528311.01 & 12.3 \\
\hline $\mathbf{P}_{11}$ & 915001.30 & 529102.01 & 19.36 \\
\hline $\mathbf{P}_{12}$ & 935000.10 & 543332.1 & 14.80 \\
\hline
\end{tabular}

\section{RESULTS AND DISCUSSIONS}

The optimization procedure gives the optimal observational weights that can be grouped into significant and zero or insignificant weights. In the proposed mathematical model, we do not allow the values of $\Delta \mathrm{p}$ greater than zero, this condition prevents that the final weights to exceed the initial weights. The significant weights, some of which may be smaller than the maximum achievable weights, are then all replaced by their corresponding maximum achievable weights. The baselines that obtained zero or insignificant weights represent those that should be deleted from the final observing plan.

After applying the optimization modelling and deleting some baselines from the final scheme, one can note that, the design matrix (observational plan) will be changed. So, the mathematical model will resolve the problem and calculate the obtained precision. In case the obtained precision is not stisfying, the new computer programm will allow adding some baselines (maximum insignificant weights).

Table 3, Comparison between the required and achievable precisions of the coordinate components after optimization

\begin{tabular}{|c|c|c|c|c|c|c|}
\hline \multirow{2}{*}{ Points } & \multicolumn{3}{|c|}{ Initial precision } & \multicolumn{3}{|c|}{ Achievable precision } \\
\hline & $\sigma_{x}(\mathrm{~mm})$ & $\sigma_{y}(\mathrm{~mm})$ & $\sigma_{z}(\mathrm{~mm})$ & $\sigma_{x}(\mathrm{~mm})$ & $\sigma_{y}(\mathrm{~mm})$ & $\sigma_{z}(\mathrm{~mm})$ \\
\hline $\mathrm{P}_{1}$ & Fixed & Fixed & Fixed & 0 & 0 & 0 \\
\hline$P_{2}$ & 7.16 & 7.16 & 7.16 & 23.09 & 23.09 & 23.09 \\
\hline$P_{3}$ & 5.56 & 5.56 & 5.56 & 13.99 & 13.99 & 13.99 \\
\hline $\mathbf{P}_{4}$ & 5.48 & 5.48 & 5.48 & 13.19 & 13.19 & 13.19 \\
\hline$P_{5}$ & 4.76 & 4.76 & 4.76 & 12.00 & 12.00 & 12.00 \\
\hline$P_{6}$ & 5.31 & 5.31 & 5.31 & 18.21 & 18.21 & 18.21 \\
\hline $\mathrm{P}_{7}$ & 4.78 & 4.78 & 4.78 & 11.24 & 11.24 & 11.24 \\
\hline $\mathbf{P}_{8}$ & Fixed & Fixed & Fixed & 0 & 0 & 0 \\
\hline$P_{9}$ & 6.30 & 6.30 & 6.30 & 16.13 & 16.13 & 16.13 \\
\hline$P_{10}$ & 5.62 & 5.62 & 5.62 & 14.63 & 14.63 & 14.63 \\
\hline$P_{11}$ & 6.44 & 6.44 & 6.44 & 15.53 & 15.53 & 15.53 \\
\hline$P_{12}$ & 8.91 & 8.91 & 8.91 & 22.62 & 22.62 & 22.62 \\
\hline
\end{tabular}

Table 4, The deleted baselines by the optimization procedure

\begin{tabular}{||c|c|c|c||c|c|c|c||}
\hline \multirow{2}{*}{ No. } & \multicolumn{2}{|c|}{ Baseline } & \multirow{2}{*}{$\begin{array}{c}\text { Distance } \\
(\mathbf{k m})\end{array}$} & \multirow{2}{*}{ No. } & \multicolumn{2}{|c|}{ Baseline } & \multirow{2}{*}{$\begin{array}{c}\text { Distance } \\
(\mathbf{k m})\end{array}$} \\
\cline { 2 - 3 } & From & To & & & From & To & \\
\hline 1 & 1 & 4 & 15.798 & 15 & 3 & 8 & 20.422 \\
\hline 2 & 1 & 5 & 17.496 & 16 & 4 & 10 & 21.861 \\
\hline 3 & 1 & 6 & 23.826 & 17 & 4 & 11 & 29.395 \\
\hline 4 & 1 & 7 & 27.109 & 18 & 5 & 10 & 21.140 \\
\hline 5 & 1 & 9 & 31.486 & 19 & 5 & 11 & 20.642 \\
\hline 6 & 1 & 10 & 35.201 & 20 & 6 & 7 & 10.740 \\
\hline 7 & 2 & 3 & 25.922 & 21 & 6 & 8 & 17.650 \\
\hline 8 & 2 & 4 & 11.219 & 22 & 6 & 9 & 29.573 \\
\hline 9 & 2 & 5 & 24.802 & 23 & 6 & 10 & 22.633 \\
\hline 10 & 2 & 6 & 32.548 & 24 & 6 & 11 & 16.974 \\
\hline 11 & 2 & 9 & 21.501 & 25 & 7 & 9 & 20.293 \\
\hline 12 & 2 & 10 & 31.667 & 26 & 9 & 10 & 13.986 \\
\hline 13 & 3 & 6 & 11.717 & 27 & 10 & 12 & 16.178 \\
\hline 14 & 3 & 7 & 18.532 & 28 & 11 & 12 & 24.545 \\
\hline
\end{tabular}


The optimization results are listed in tables (3) and (4). first, The standard deviations of the station coordinates as obtained from the optimized observing scheme is listed in Table (3), while Table (4) gives the deleted baselines by optimization procedure. Figure (7) shows The optimized observing scheme. from which the following can be observed:

1. The standard deviations of the net points before applying the optimization model ranges between $(4.76) \mathrm{mm}$ at point $\mathrm{P}_{5}$ and $(8.91) \mathrm{mm}$ at point $\mathrm{P}_{12}$. The standard deviations of the net points after applying the optimization model ranges between (11.24) $\mathrm{mm}$ at point $\mathrm{P}_{7}$ and (23.09) $\mathrm{mm}$ at point $\mathrm{P}_{2}$. So, all standard deviations of the coordinate components still less than the required value $(25 \mathrm{~cm})$.

2. Using the optimization model, deleting 28 baselines out of a total of 65 baselines while adhering to the required precision resulted in that saving $43.08 \%$ of field work.

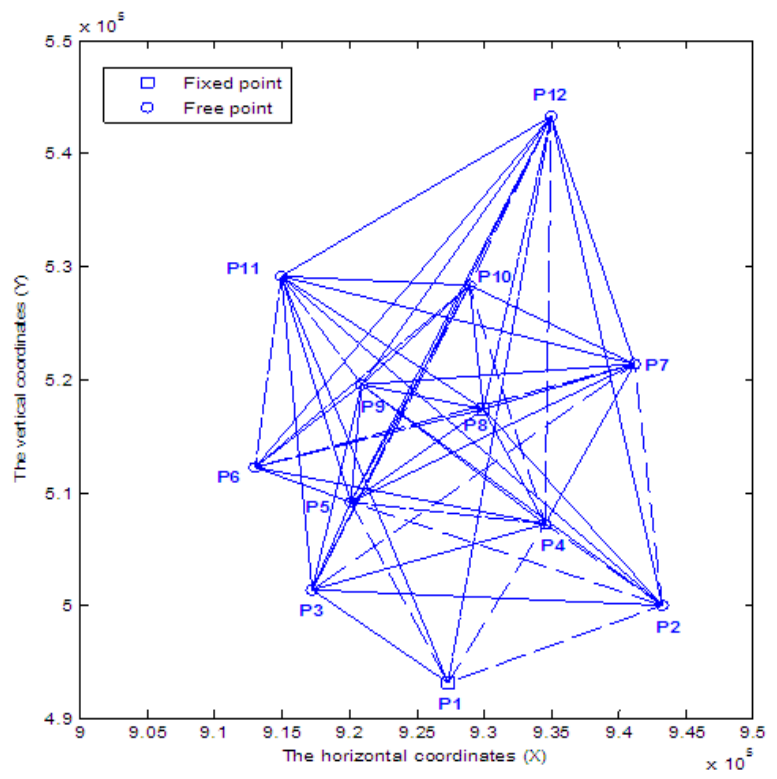

Fig. 7 The optimized observation scheme

\section{CONCLUSIONS AND FUTURE WORK}

The optimization procedure gives the optimal observational weights that can be grouped into significant and zero or insignificant weights. In the proposed mathematical model, we do not allow the values of $\Delta \mathrm{p}$ greater than zero, this condition prevents that the final weights to exceed the initial weights. The significant weights, some of which may be smaller than the maximum achievable weights, are then all replaced by their corresponding maximum achievable weights. The baselines that obtained zero or insignificant weights represent those that should be deleted from the final observing plan.

\section{REFRENCES}

[1] Balasubramaniam P., Kumar A.V.A. (2009). "Solution of Matrix Riccati Differential Equation for Nonlinear Singular System Using Genetic Programming”. Genet Program Evoluable Mach, 10, pp. 71-89.

[2] Berné J.L., Baselga S. (2004). "First-order Design of Geodetic Networks Using the Simulated Annealing Method". Journal of Geodesy, 78, pp. 47-54.

[3] Chih-Hung Wu., Hung-Ju C., Wei-Han Su.(2008)."Direct Transformation of Coordinates for GPS Positioning Using the Techniques of Genetic Programming and Symbolic Regression”. Engineering Applications of Artificial Intelligence, 21, pp. 1347-1359.

[4] Deb K. (1996). "GAs and Soft Computing: GAs for Function Optimization". Physica-Verlag, 329.

[5] Doma M.I. (2004). "Application Of Optimization Theory To Geodetic Networks Using Different Orders Design Problems" M. Sc. Thesis at the Faculty of Engineering, Minoufiya University, Minoufiya, Egypt.

[6] Doma M.I. (2008). “The Optimal Improvement of Geodetic Network Using Interactive Graphics System". Doctoral Thesis at the Faculty of Engineering, Minoufiya University, Minoufiya, Egypt.

[7] Ekland S.K. (2004). "A Massively Parallel Architecture for Distributed Genetic Algorithms". Parallel Computing, 30, pp. 647676.

[8] Elshouny A.F. (2008). "The Combined Adjustment of Terrestrial and Satellite Control Network". M. Sc. Thesis at the Faculty of Engineering, Minoufiya University, Minoufiya, Egypt.

[9] Fidanova S., Alba E., and Molina G. (2010). "Hybrid ACO Algorithm for the GPS Surveying Problem". Springer-Verlag Berlin Heidelberg, pp. 318-325.

[10] Kuang, S.L. (1994). "A strategy for GPS survey planning: Choice of optimum baselines" Proceedings of the 1994 ASPRS/ACSM Annual Convention \& Exposition, Technical papers, Vol. 2, pp. 42-58, Reno, Nevada, April 25-28.

[11] Melanie M. (1999). "An Introduction to Genetic Algorithms". Cambridge, Massachusetts, London, England, Fifth Printing.

[12] Ponce-Cruz P., Ramirez-Figueroa F.D. (2010). "Intelligent Control Systems with LabVIEW $^{\mathrm{TM}}$,. Springer, 2010. 
[13] Riesberg L. (2003). "Genetic Algorithms". New Tools for the Programmers' Toolbox, April 16, 2003.

[14] Saleh H.A., Dare P. (2001). "Effective Heuristics for the GPS Survey Network of Malta: Simulated Annealing and Tabu Search Technique". Journal of Heuristics, 7, pp. 533549.@ Kluwer Academic Publishers. Manufactured in the Netherlands.

[15] Saleh H. A. and Chelouah R. (2004). " The Design of the Global Navigation Satellite System Surveying Networks Using Genetic Algorithm." Engineering Applications of Artificial Intelligence, 17 , p. 111-122.

[16] Sivanandam S. N. and Deepa S.N. (2008). "Introduction to Genetic Algorithms". Springer-Verlag Berlin Heidelberg.

[17] Teke K., Yalcinkaya M., and Konak H., (2008). "Optimization of GPS Networks for Landslide Areas". Fresenius Environmental Bulletin, 17, pp. 264-276.

[18] Weise T. (2009). "Global Optimization Algorithms". Theory and Application, June 26, 2009.
[19] Sahabi H., Rajabi M. A. and Blais (2008). "Optimal Configuration Design of Geodetic Networks Using Penalty Function-Based Genetic Algorithm, Geophysical Research Abstracts, vol. 10, EGU2008-A-10202, 2008.

[20] Saleh H. A. and Chelouah R. (2004). " The Design of the Global Navigation Satellite System Surveying Networks Using Genetic Algorithm." Engineering Applications of Artificial Intelligence, 17 (2004) 111-122.

[21] Schaffrin, B. (1981): "Some proposal concerning the diagonal Second Order Design of geodetic networks." Manuscripta Geodetica, Vol. 6, No. 3, p. 303-326.

[22] Sivanandam S. N. and Deepa S.N. (2008). "Introduction to Genetic Algorithms". Springer-Verlag Berlin Heidelberg, 2008.

[23] Olague, G. (2001): “Autonomous Photogrammetric Network Design Using Genetic Algorithms" Evo Workshop 2001, LNCS 2037, pp. 353-363 , 2001 , SpringVerlag Berlin Heidelberg 2001. 